\title{
Erratum: Fevers in Adult Lupus Patients
}

Homa Timlin ${ }^{1}$, Abrahim Syed ${ }^{2}$, Uzma Haque ${ }^{3}$, Brittany Adler ${ }^{3}$, Genevieve Law ${ }^{4}$, Kirthi Machireddy ${ }^{5}$, Rebecca Manno ${ }^{3}$

1. Medicine, The Johns Hopkins University School of Medicine 2. Rheumatology, The Johns Hopkins University School of Medicine 3. Division of Rheumatology, The Johns Hopkins University School of Medicine 4. Rheumatology, FETCH South Iseland 5. Other, University of the Sciences In Philadelphia

$\square$ Corresponding author: Homa Timlin, htimlin1@jhmi.edu

Disclosures can be found in Additional Information at the end of the article

Corresponding author: Homa Timlin

1. Medicine, The Johns Hopkins University School of Medicine 2. Rheumatology, The Johns Hopkins University School of Medicine 3. Division of Rheumatology, The Johns Hopkins University School of Medicine 4. Division of Rheumatology, The Johns Hopkins University School of Medicine 5. Rheumatology, FETCH South Iseland 6. Other, University of the Sciences In Philadelphia 7. Division of Rheumatology, The Johns Hopkins University School of Medicine

How to cite this erratum

Timlin H, Syed A, Haque U, et al. (May 01, 2018) Correction: Fevers in Adult Lupus Patients. Cureus 10(5): c12. doi:10.7759/cureus.c12

\section{Correction Notice}

The following funding acknowledgement was erroneously omitted from the original published article:

BA was supported by the National Institute of Arthritis and Musculoskeletal and Skin Diseases of the National Institute of Health under Award Number T32AR048522. 